\title{
Coefficient of normal restitution of viscous particles and cooling rate of granular gases
}

\author{
Thomas Schwager因 and Thorsten Pösche团 \\ Humboldt-Universität zu Berlin, Institut für Physik, \\ Invalidenstraße 110, D-10115 Berlin, Germany
}

(October 11, 2018)

\begin{abstract}
We investigate the cooling rate of a gas of inelastically interacting particles. When we assume velocity dependent coefficients of restitution the material cools down slower than with constant restitution. This behavior might have large influence to clustering and structure formation processes.
\end{abstract}

PACS numbers: 83.70.Fn, 62.40.+i, 81.40.Lm, 05.40. -y, $46.10+\mathrm{z}$

Typeset using REVTEX 
The behavior of granular gases has been of large scientific interest in recent time. Goldhirsch and Zanetti [1] and McNamara and Young [2] have shown that a homogeneous granular gas is unstable. After some time one observes dense regions (clusters) and voids. To evaluate the loss of mechanical energy due to collisions one introduces the coefficient of (normal) restitution

$$
g^{\prime}=\epsilon g,
$$

where $g=|\vec{g}|$ and $g^{\prime}=\left|\vec{g}^{\prime}\right|$, describing the loss of relative normal velocity $g^{\prime}$ of a pair of colliding particles after the collision with respect to the impact velocity $g$

It can be shown that even for three particles for certain region of the coefficient of restitution there exist initial conditions which lead to a behavior which is called "inelastic collapse". This means that the particles accomplish an infinite number of collisions in finite time [2]. The conditions under which one can observe inelastic collapse have been studied in one dimensional systems [3] as well as in higher dimension [4]. Recently it was shown numerically that the probability for a collapse rises significantly when the particles have rotational degree of freedom [5]. In this case the collapse is possible for much larger coefficients of restitution than for non rotating particles. Other interesting related results concern bouncing ball experiments on vibrating tables where complicated dynamical behavior is observed (e.g. [6]). Recently complicated, under certain circumstances irregular motion of a bouncing cantilever of an atomic force microscope when excited by a transducer was investigated [7].

In the investigations [1] the approximation of constant coefficient of restitution was assumed. Solving viscoelastic equations for spheres currently it was shown that the coefficient of normal restitution $\epsilon$ is not a constant but a function of the impact velocity $\epsilon(g)$ itself [8,9]. For the "compression" $\xi=R_{1}+R_{2}-\left|\vec{r}_{1}-\vec{r}_{2}\right|$ of particles with radii $R_{1}$ and $R_{2}$ at positions $\vec{r}_{1}$ and $\vec{r}_{2}$ one finds

$$
\begin{gathered}
\ddot{\xi}+\rho\left(\xi^{3 / 2}+\frac{3}{2} A \sqrt{\xi} \dot{\xi}\right)=0 \\
\rho=\frac{2 Y \sqrt{R^{\mathrm{eff}}}}{3 m^{\mathrm{eff}}\left(1-\nu^{2}\right)}
\end{gathered}
$$


$Y$ is the Young modulus, $\nu$ the Poisson ratio and

$$
\begin{aligned}
m_{\mathrm{eff}} & =\frac{m_{1} m_{2}}{m_{1}+m_{2}} \\
R_{\mathrm{eff}} & =\frac{R_{1} R_{2}}{R_{1}+R_{2}}
\end{aligned}
$$

are the effective radius and mass of the grains, respectively. $A$ is a material constant depending on the Young modulus, the viscous constants and the Poisson ratio of the material. Equation (2) was derived under the precondition that the colliding spheres have impact velocity much less than speed of sound in the particle material. For details see [8]. The initial conditions for solving (2) are

$$
\begin{aligned}
& \xi(0)=0 \\
& \dot{\xi}(0)=g .
\end{aligned}
$$

The coefficient of restitution $\epsilon$ of at time $t=0$ colliding spherical grains can be found from this equation relating the relative normal velocities $g=\dot{\xi}(0)$ at time of impact and at time $t_{c}$, when the particles separate after the collision, i.e. $t_{c}$ is the collision time:

$$
\epsilon=-\dot{\xi}\left(t_{c}\right) / \dot{\xi}(0)
$$

The (numerical) integration of equation (6) yields the coefficient of restitution as a function of the impact velocity (see fig. 1 in [8]) which is in good agreement with experimental data [10]. Constant coefficient of restitution, however, does not agree with experimental experience [11]. Other theoretical work on this topic can be found e.g. in [12,13].

Consider a gas of granular particles at a given initial granular Temperature $T_{0}$. Then the question arises how the temperature decreases with time due to inelastic collisions. This problem has been investigated earlier [14,15] for the case of constant coefficient of restitution and the result is (s. also [16])

$$
T(t)=T_{0}(1+t / \tau)^{-2}
$$

The time scale $\tau$ is a material constant. The temperature decay (7) is the origin of the cluster instabilities which have been investigated recently [1,2]. 
The aim of the present paper is to derive an explicite analytic expression for the coefficient of normal restitution $\epsilon(g)$ as a function of the impact velocity $g$. A direct consequence of this result will be a refined expression for the temperature decay of a granular gas.

The duration of collision $t_{c}^{0}$ for the undamped problem $(A=0)$ is given by [17]:

$$
t_{c}^{0}=\frac{\Theta_{c}^{0}}{\rho^{\frac{2}{5}} g^{\frac{1}{5}}}
$$

We want to point out here that $\Theta_{c}^{0}$ is a constant pure number, not depending on any material properties. Hence, $t_{c}^{0}$ depends only on the material constant $\rho$ and on the initial velocity $g$. We use equation (8) to define a rescaled dimensionless time $\Theta$ :

$$
\Theta=\rho^{\frac{2}{5}} g^{\frac{1}{5}} t
$$

Using the abbreviations

$$
\begin{aligned}
v & =\rho^{2} g \\
\alpha & =\frac{3}{2} A
\end{aligned}
$$

and a new set of variables

$$
\begin{aligned}
\Theta & =\rho^{\frac{2}{5}} g^{\frac{1}{5}} t=v^{\frac{1}{5}} t \\
x(\Theta) & =\rho^{2} \xi(t)
\end{aligned}
$$

we rewrite (2) in the form

$$
\ddot{x}+\alpha v^{-\frac{1}{5}} \dot{x} \sqrt{x}+v^{-\frac{2}{5}} x^{\frac{3}{2}}=0
$$

with $\dot{x}=\frac{d}{d \Theta} x$. We see that

$$
\frac{d x}{d t}(0)=\frac{1}{\rho^{2}} \frac{d \xi}{d t}(0)=\frac{g}{\rho^{2}}=v=v^{\frac{1}{5}} \frac{d x}{d \Theta}(0)
$$

Hence the initial conditions in our new variables $x$ and $\Theta$ read

$$
\begin{aligned}
x(0) & =0 \\
\frac{d x}{d \Theta}(0)=\dot{x}(0) & =v^{\frac{4}{5}}
\end{aligned}
$$


Both equations of motion, (2) and (12) become special at $x=0$ or $\xi=0$, respectively, i.e. all derivatives of third order and higher diverge. This will be shown for the case of $x$ :

$$
\begin{aligned}
\frac{d}{d \Theta} \ddot{x} & =-\frac{d}{d \Theta}\left(\alpha v^{-\frac{1}{5}} \dot{x} \sqrt{x}+v^{-\frac{2}{5}} x^{\frac{3}{2}}\right) \\
& =\alpha v^{-\frac{1}{5}}\left(\ddot{x} \sqrt{x}+\frac{\dot{x}}{2 \sqrt{x}}\right)-\frac{3}{2} v^{-\frac{2}{5}} \dot{x} \sqrt{x}
\end{aligned}
$$

Hence

$$
\lim _{x \rightarrow 0} \frac{d^{3}}{d \Theta^{3}} x= \pm \infty
$$

and so are the higher derivatives. Because of this singularity we must not expand $x$ in powers of $\Theta$. Because of the initial conditions $x(\Theta)$ has the form

$$
\begin{aligned}
& x(\Theta)=v^{\frac{4}{5}} \Theta(1+\eta(\Theta)) \\
& \eta(0)=0,
\end{aligned}
$$

which defines the function $\eta(\Theta)$. Using transformation (17) we find

$$
\Theta \ddot{\eta}+2 \dot{\eta}+\alpha v^{\frac{1}{5}} \Theta^{\frac{3}{2}} \dot{\eta} \sqrt{1+\eta}+\left(\alpha v^{\frac{1}{5}} \sqrt{\Theta}+\Theta^{\frac{3}{2}}\right)(1+\eta)^{\frac{3}{2}}=0
$$

In (19) occur terms $\Theta^{0.5}$ and $\Theta^{1.5}$, therefore we expand $\eta$ in powers of $\sqrt{\Theta}$

$$
\eta=\sum_{k=0}^{\infty} a_{k} \Theta^{\frac{k}{2}}
$$

The first coefficient $a_{0}$ vanishes because of the initial condition for $x$. When we require

$$
\dot{\eta}=\frac{a_{1}}{2 \sqrt{\Theta}}+a_{2}+\ldots
$$

to be finite at $\Theta=0$ the second coefficient $a_{1}$ must vanish as well. With Taylor expansion of $\sqrt{1+\eta}$ and $(1+\eta)^{\frac{3}{2}}$ for small $\eta$ we arrive at

$$
\eta=-\frac{4}{15} \alpha v^{\frac{1}{5}} \Theta^{\frac{3}{2}}-\frac{4}{35} \Theta^{\frac{5}{2}}+\frac{3}{70} \alpha v^{\frac{1}{5}} \Theta^{4}+\frac{1}{15} \alpha^{2} v^{\frac{2}{5}} \Theta^{3} \ldots
$$

and therefore 


$$
\begin{aligned}
x= & v^{\frac{4}{5}} \Theta-\frac{4}{15} \alpha v \Theta^{\frac{5}{2}}-\frac{4}{35} v^{\frac{4}{5}} \Theta^{\frac{7}{2}}+\frac{1}{15} \alpha^{2} v^{\frac{6}{5}} \Theta^{4} \\
& +\frac{3}{70} \alpha v \Theta^{5}-\frac{38}{2475} \alpha^{3} v^{\frac{7}{5}} \Theta^{\frac{11}{2}}+\frac{1}{175} v^{\frac{4}{5}} \Theta^{6}+\ldots
\end{aligned}
$$

Rearranging the full series (23) one finds

$$
\begin{aligned}
x & =v^{\frac{4}{5}}\left(\Theta-\frac{4}{35} \Theta^{\frac{7}{2}}+\frac{1}{175} \Theta^{6}+\ldots\right) \\
& +\alpha v\left(-\frac{4}{15} \Theta^{\frac{5}{2}}+\frac{3}{70} \Theta^{5}+\ldots\right) \\
& +\alpha^{2} v^{\frac{6}{5}}\left(\frac{1}{15} \Theta^{4}+\ldots\right)+\ldots \\
& =v^{\frac{4}{5}} x_{0}(\Theta)+\alpha v x_{1}(\Theta)+\alpha^{2} v^{\frac{6}{5}} x_{2}(\Theta) \ldots
\end{aligned}
$$

$v^{\frac{4}{5}} x_{0}$ is the solution of the undamped (elastic) collision (s. dashed line in fig. 目). The full line in fig. 1 shows the damped motion according to eq. (23). The direct numerical integration of eq. (12) collapses with the full line.

For $x\left(\frac{1}{2} \Theta_{c}^{0}\right)$ where $\Theta_{c}^{0}$ is the duration of the undamped collision one finds using (24):

$$
\begin{aligned}
x\left(\frac{\Theta_{c}^{0}}{2}\right) & =v^{\frac{4}{5}} x_{0}\left(\frac{\Theta_{c}^{0}}{2}\right)+\alpha v x_{1}\left(\frac{\Theta_{c}^{0}}{2}\right)+\alpha^{2} v^{\frac{6}{5}} x_{2}\left(\frac{\Theta_{c}^{0}}{2}\right)+\ldots \\
& =v^{\frac{4}{5}} B_{0}+\alpha v B_{1}+\alpha^{2} v^{\frac{6}{5}} B_{2}+\ldots
\end{aligned}
$$

which we do not need now but later on.

Note that the coefficients $B_{k}$ are constants, i.e. they do not depend on $v$ nor on material constants.

Equations (21) and (12), respectively, hold for the entire collision. The collision starts with $v$ and ends with $v^{\prime}$. For practical purposes we now define the term inverse collision. The inverse collision is a collision which starts at time $\Theta_{c}$ with relative velocity $v^{\prime}$ and ends at time 0 with relative velocity $v$, i.e. time runs in inverse direction during the inverse collision. The equation of motion for $x^{i n v}$, i.e. for a collision in inverse time follows from (12). Since the inverse collision starts with $v^{\prime}$ we have to replace $v$ by $v^{\prime}$. Because of the time reversal we have to change the sign of time derivatives of odd orders, i.e. $\dot{x} \rightarrow-\dot{x}^{i n v}$. The equation of motion for the inverse collision reads 


$$
\ddot{x}^{i n v}-\alpha\left(v^{\prime}\right)^{-\frac{1}{5}} \dot{x}^{i n v} \sqrt{x^{i n v}}+\left(v^{\prime}\right)^{-\frac{2}{5}}\left(x^{i n v}\right)^{\frac{3}{2}}=0
$$

A motion due to eq. (26) in normal time would be an accelerated one. However, we shall mention here that eqs. (12) and (26) describe strictly the same physical motion. The solution $x^{i n v}$ of the inverse problem can be derived from the solution of the direct problem replacing $\alpha \rightarrow-\alpha$ and $v \rightarrow v^{\prime}$.

$$
x^{i n v}\left(\Theta^{\prime}\right)=\left(v^{\prime}\right)^{\frac{4}{5}} x_{0}\left(\Theta^{\prime}\right)-\alpha v^{\prime} x_{1}\left(\Theta^{\prime}\right)+\alpha^{2}\left(v^{\prime}\right)^{\frac{6}{5}} x_{2}\left(\Theta^{\prime}\right) \ldots
$$

Now we determine the collision time $\Theta_{c}$ and the final velocity. One direct method to calculate $\Theta_{c}$ would be to determine the solution of $x(\Theta)=0$ using Taylor expansion of $x$ in the region close to $\Theta_{c}^{0}$. It can be seen easily that this method fails since all derivatives of $\frac{d^{n}}{d \Theta^{n}} x$ with $n \geq 3$ diverge for $\Theta=\Theta_{c}^{0}$. Therefore $\Theta_{c}$ has to be calculated by an indirect methode.

The problem will be subdivided into two parts (s. fig. 2):

a) the motion of the particles $x$ from $\Theta=0$ to time $\Theta_{m}$ when $x$ approaches its maximum and where $\dot{x}$ changes its sign, and

b) from $\Theta_{m}$ to $\Theta_{c}$.

In case of undamped motion where $\alpha=0$ we have $\Theta_{m}=\Theta_{c}^{0} / 2$. In part b) we do not consider the collision itself but the inverse problem in the interval $\left(\Theta=0, \Theta_{m}^{\prime}\right)$, with $\Theta_{m}^{\prime}$ being the time where $x^{i n v}$ approaches its maximum. The continuity of both parts means $x\left(\Theta_{m}\right)=x^{i n v}\left(\Theta_{m}^{\prime}\right)$.

For finite damping $\alpha \neq 0$ we write $\Theta_{m}=\Theta_{c}^{0} / 2+\delta$ and $\Theta_{m}^{\prime}=\left(\Theta_{c}^{0}\right)^{\prime} / 2+\delta^{\prime}$ and remind that $\Theta_{c}^{0}=\left(\Theta_{c}^{0}\right)^{\prime}$. To get an expression for $\delta$ we expand

$$
\begin{aligned}
\dot{x}\left(\frac{\Theta_{c}^{0}}{2}+\delta\right)=0= & \dot{x}\left(\frac{\Theta_{c}^{0}}{2}\right)+\delta \ddot{x}\left(\frac{\Theta_{c}^{0}}{2}\right)+\frac{\delta^{2}}{2} \frac{d^{3}}{d \Theta^{3}} x\left(\frac{\Theta_{c}^{0}}{2}\right)+\ldots \\
= & v^{\frac{4}{5}}\left(\dot{x}_{0}\left(\frac{\Theta_{c}^{0}}{2}\right)+\delta \ddot{x}_{0}\left(\frac{\Theta_{c}^{0}}{2}\right)+\frac{\delta^{2}}{2} \frac{d^{3}}{d \Theta^{3}} x_{0}\left(\frac{\Theta_{c}^{0}}{2}\right)+\ldots\right) \\
& +v \alpha\left(\dot{x}_{1}\left(\frac{\Theta_{c}^{0}}{2}\right)+\delta \ddot{x}_{1}\left(\frac{\Theta_{c}^{0}}{2}\right)+\frac{\delta^{2}}{2} \frac{d^{3}}{d \Theta^{3}} x_{1}\left(\frac{\Theta_{c}^{0}}{2}\right)+\ldots\right) \\
& +v^{\frac{6}{5}} \alpha^{2}\left(\dot{x}_{2}\left(\frac{\Theta_{c}^{0}}{2}\right)+\delta \ddot{x}_{2}\left(\frac{\Theta_{c}^{0}}{2}\right)+\frac{\delta^{2}}{2} \frac{d^{3}}{d \Theta^{3}} x_{2}\left(\frac{\Theta_{c}^{0}}{2}\right)+\ldots\right)
\end{aligned}
$$


and using $\dot{x}_{0}\left(\Theta_{c}^{0} / 2\right)=0\left(v^{\frac{4}{5}} x_{0}\right.$ is the solution of the undamped problem $)$

$$
\delta=-\alpha v^{\frac{1}{5}} \frac{\dot{x}_{1}\left(\Theta_{c}^{0} / 2\right)}{\ddot{x}_{0}\left(\Theta_{c}^{0} / 2\right)}+\mathcal{O}\left(\alpha^{2}\right)
$$

The expression (30) has to be inserted into the Taylor expansion of $x\left(\Theta_{c}^{0} / 2+\delta\right)$ :

$$
\begin{aligned}
x\left(\Theta_{c}^{0} / 2+\delta\right)= & v^{\frac{4}{5}}\left(x_{0}\left(\frac{\Theta_{c}^{0}}{2}\right)+\delta \dot{x}_{0}\left(\frac{\Theta_{c}^{0}}{2}\right)+\frac{\delta^{2}}{2} \ddot{x}_{0}\left(\frac{\Theta_{c}^{0}}{2}\right)+\ldots\right) \\
& +\alpha v\left(x_{1}\left(\frac{\Theta_{c}^{0}}{2}\right)+\delta \dot{x}_{1}\left(\frac{\Theta_{c}^{0}}{2}\right)+\frac{\delta^{2}}{2} \ddot{x}_{1}\left(\frac{\Theta_{c}^{0}}{2}\right)+\ldots\right) \\
= & v^{\frac{4}{5}} x_{0}\left(\frac{\Theta_{c}^{0}}{2}\right)+\alpha v x_{1}\left(\frac{\Theta_{c}^{0}}{2}\right)-\frac{\alpha^{2} v^{\frac{6}{5}}}{2} \frac{\dot{x}_{1}^{2}\left(\Theta_{c}^{0} / 2\right)}{\ddot{x}_{0}\left(\Theta_{c}^{0} / 2\right)} \\
& +\alpha^{2} v^{\frac{6}{5}} x_{2}\left(\frac{\Theta_{c}^{0}}{2}\right)+\mathcal{O}\left(\alpha^{3}\right) .
\end{aligned}
$$

Hence

$$
x\left(\Theta_{m}\right)=v^{\frac{4}{5}} x_{0}\left(\Theta_{c}^{0} / 2\right)+\alpha v x_{1}\left(\Theta_{c}^{0} / 2\right)+\alpha^{2} v^{\frac{6}{5}}\left(x_{2}\left(\frac{\Theta_{c}^{0}}{2}\right)-\frac{1}{2} \frac{\dot{x}_{1}^{2}\left(\Theta_{c}^{0} / 2\right)}{\ddot{x}_{0}\left(\Theta_{c}^{0} / 2\right)}\right)+\ldots
$$

Replacing again $v \rightarrow v^{\prime}$ and $\alpha \rightarrow-\alpha$ yields

$$
\begin{aligned}
\delta^{\prime} & =\alpha\left(v^{\prime}\right)^{\frac{1}{5}} \frac{\dot{x}_{1}\left(\Theta_{c}^{0} / 2\right)}{\ddot{x}_{0}\left(\Theta_{c}^{0} / 2\right)}+\mathcal{O}\left(\alpha^{2}\right) \\
x^{i n v}\left(\Theta_{m}^{\prime}\right) & =\left(v^{\prime}\right)^{\frac{4}{5}} x_{0}\left(\Theta_{c}^{0} / 2\right)-\alpha v^{\prime} x_{1}\left(\Theta_{c}^{0} / 2\right)+\alpha^{2}\left(v^{\prime}\right)^{\frac{6}{5}}\left(x_{2}\left(\frac{\Theta_{c}^{0}}{2}\right)-\frac{1}{2} \frac{\dot{x}_{1}^{2}\left(\Theta_{c}^{0} / 2\right)}{\ddot{x}_{0}\left(\Theta_{c}^{0} / 2\right)}\right)+\ldots
\end{aligned}
$$

As explained above both solutions (33) and (35) have to be equal. With

$$
\beta=x_{2}\left(\frac{\Theta_{c}^{0}}{2}\right)-\frac{1}{2} \frac{\dot{x}_{1}^{2}\left(\Theta_{c}^{0} / 2\right)}{\ddot{x}_{0}\left(\Theta_{c}^{0} / 2\right)}
$$

we write

$$
v^{\frac{4}{5}} x_{0}\left(\frac{\Theta_{0}^{c}}{2}\right)+\alpha v x_{1}\left(\frac{\Theta_{0}^{c}}{2}\right)+\alpha^{2} v^{\frac{6}{5}} \beta=\left(v^{\prime}\right)^{\frac{4}{5}} x_{0}\left(\frac{\Theta_{0}^{c}}{2}\right)-\alpha v^{\prime} x_{1}\left(\frac{\Theta_{0}^{c}}{2}\right)+\alpha^{2}\left(v^{\prime}\right)^{\frac{6}{5}} \beta
$$

We expand $v^{\prime}$ in $\alpha$

$$
v^{\prime}=v+\alpha v_{1}+\alpha^{2} v_{2}+\ldots,
$$

and find 


$$
\begin{aligned}
& v^{\frac{4}{5}} x_{0}\left(\frac{\Theta_{0}^{c}}{2}\right)+\alpha v x_{1}\left(\frac{\Theta_{0}^{c}}{2}\right)+\alpha^{2} v^{\frac{6}{5}} \beta \\
= & v^{\frac{4}{5}}\left(1+\frac{\delta v}{v}\right)^{\frac{4}{5}} x_{0}\left(\frac{\Theta_{0}^{c}}{2}\right)-\alpha v\left(1+\frac{\delta v}{v}\right) x_{1}\left(\frac{\Theta_{0}^{c}}{2}\right)+\alpha^{2} v^{\frac{6}{5}}\left(1+\frac{\delta v}{v}\right)^{\frac{6}{5}} \beta
\end{aligned}
$$

with $\delta v=\alpha v_{1}+\alpha^{2} v_{2}+\ldots$. Writing $\left(1+\frac{\delta v}{v}\right)^{\frac{n}{5}}$ in powers of $\alpha$ and comparing coefficients yields finally

$$
\begin{aligned}
v^{\prime} & =v\left(1+\frac{5}{2} \alpha v^{\frac{1}{5}} \frac{x_{1}\left(\frac{\Theta_{c}^{0}}{2}\right)}{x_{0}\left(\frac{\Theta_{c}^{0}}{2}\right)}+\frac{15}{4} \alpha^{2} v^{\frac{2}{5}}\left(\frac{x_{1}\left(\frac{\Theta_{c}^{0}}{2}\right)}{x_{0}\left(\frac{\Theta_{c}^{0}}{2}\right)}\right)^{2}+\ldots\right) \\
& =v\left(1-\alpha v^{\frac{1}{5}} C_{1}+\alpha^{2} v^{\frac{2}{5}} C_{2}+\ldots\right),
\end{aligned}
$$

with

$$
\begin{aligned}
C_{1} & =\frac{5}{2} \frac{x_{1}\left(\frac{\Theta_{c}^{0}}{2}\right)}{x_{0}\left(\frac{\Theta_{c}^{0}}{2}\right)} \\
C_{2} & =\frac{15}{4}\left(\frac{x_{1}\left(\frac{\Theta_{c}^{0}}{2}\right)}{x_{0}\left(\frac{\Theta_{c}^{0}}{2}\right)}\right)^{2} .
\end{aligned}
$$

Since $\Theta_{c}^{0}$ does depend neither on any material properties nor on the impact velocity $g$ or $v$, respectively, $C_{1}$ and $C_{2}$ are pure numerical constants. Evaluating $C_{1}$ and $C_{2}$ in (41) numerically yields $C_{1}=1.15344$ and $C_{2}=0.79826$.

For coefficient of normal restitution one gets

$$
\begin{aligned}
\epsilon=\frac{v^{\prime}}{v} & =1-\alpha v^{\frac{1}{5}} C_{1}+\alpha^{2} v^{\frac{2}{5}} C_{2}+\ldots \\
& =1-C_{1} A \rho^{\frac{2}{5}} g^{\frac{1}{5}}+C_{2} A^{2} \rho^{\frac{4}{5}} g^{\frac{2}{5}}+\ldots
\end{aligned}
$$

with $g$ being the impact velocity (fig [3). For the duration of collision we find with (30), (35) and (40)

$$
\begin{aligned}
t_{c} & =\left(\frac{\Theta_{c}^{0}}{2}+\delta\right) v^{-\frac{1}{5}}+\left(\frac{\Theta_{c}^{0}}{2}+\delta^{\prime}\right)\left(v^{\prime}\right)^{-\frac{1}{5}} \\
& =\Theta_{c}^{0} v^{-\frac{1}{5}}\left(1-\frac{1}{4} \alpha v^{\frac{1}{5}} \frac{x_{1}\left(\Theta_{c}^{0} / 2\right)}{x_{0}\left(\Theta_{c}^{0} / 2\right)}\right)+\mathcal{O}\left(\alpha^{2}\right) \\
& =\Theta_{c}^{0} v^{-\frac{1}{5}}\left(1+\frac{1}{10} C_{1} \alpha v^{\frac{1}{5}}\right)+\mathcal{O}\left(\alpha^{2}\right) \\
& =\Theta_{c}^{0} \rho^{-\frac{2}{5}} g^{-\frac{1}{5}}\left(1+\frac{1}{10} C_{1} \alpha \rho^{\frac{2}{5}} g^{\frac{1}{5}}\right)+\mathcal{O}\left(\alpha^{2}\right) .
\end{aligned}
$$




$$
\begin{aligned}
\Theta_{c} & =v^{\frac{1}{5}} t_{c} \\
& =\Theta_{c}^{0}\left(1+\frac{1}{10} C_{1} \alpha v^{\frac{1}{5}}\right)+\mathcal{O}\left(\alpha^{2}\right)
\end{aligned}
$$

To check the theoretical result (eqs. 41) we integrated numerically eq. (12) and received the curves $\epsilon(v)$ and $\Theta_{c}(v)$. Then we fitted $C_{1}$ and $C_{2}$ to these data using (42) and (44). For instance for $\alpha=0.05$ we found $C_{1}^{\text {num }}=1.15356$ and $C_{2}^{\text {num }}=0.80439$ from the curve $\epsilon(v)$ (s. eq. 42). The fit of $C_{1}$ to $\Theta_{c}(v)$ (s. eq. 44) gives $C_{1}^{\text {num }}=1.15342$. For other values of $\alpha$ we found very similar numbers. Hence, the numerical results agree with theory.

When we use the velocity dependent coefficient of restitution in the collision term of Boltzmann equation

$$
\dot{T} \sim \iint d v_{1} d v_{2}\left(1-\epsilon^{2}\right)\left|v_{1}-v_{2}\right|^{3} f\left(v_{1}\right) f\left(v_{2}\right)
$$

we get the cooling rate for dissipative gas

$$
T \sim T_{0} /\left(1+\frac{t}{\tau^{\prime}}\right)^{\frac{5}{3}}
$$

Our final results eq. (42) shows that for viscoelastic colliding smooth bodies the coefficient of normal restitution is a decreasing function with rising impact velocity: $1-\epsilon \sim g^{\frac{1}{5}}$. A direct consequence is the cooling rate of a granular gas (eq. (46)): a granular gas consisting of viscoelastic particles cools down significantly slower than a gas of particles which collide with constant coefficient of restitution (s. eq. (77)). Due to our understanding it is neither self-evident whether the clustering observed in granular gases of the latter type, and the extreme case of this effect, the inelastic collapse, will change their overall behavior nor whether they exist at all. These questions should be reconsidered in detail for velocity dependent restitution.

The authors are grateful to N. Brilliantov, S. Esipov, H. Herrmann, F. Spahn and W. Young for helpful discussions, and J.-M. Hertzsch for providing relevant literature. 


\section{REFERENCES}

* Email: schwager@itp02.physik.hu-berlin.de, thorsten@itp02.physik.hu-berlin.de

[1] I. Goldhirsch and G. Zanetti, Phys. Rev. Lett., 70, 1619 (1993).

[2] S. McNamara and W. R. Young, Phys. Fluids A 5, 34 (1993); Phys. Rev. E 50, R28 (1994).

[3] P. Constantin, E. Grossmann, and M. Mungan, Physica D 83, 409 (1995); E. Grossmann and M. Mungan preprint.

[4] Y. Du, H. Li, and L. P. Kadanoff, Phys. Rev. Lett. 74, 1268 (1995); T. Zhou and L. P. Kadanoff, preprint.

[5] N. Schörghofer and T. Zhou, Phys. Rev. E 54, 5511 (1996).

[6] A. Mehta and J. M. Luck, Phys. Rev. Lett. 65, 393 (1990); J. M. Luck and A. Mehta, Phys. Rev. E 48, 3988 (1993).

[7] N. A. Burnham, A. J. Kulik, and G. Gremaud, Phys. Rev. Lett. 74, 5092 (1995).

[8] N. V. Brilliantov, F. Spahn, J.-M. Hertzsch, and T. Pöschel, Phys. Rev. E, 53, 5382 (1996).

[9] G. Kuwabara and K. Kono, Jap. J. Appl. Phys, 26, 1230 (1987).

[10] F. G. Bridges, A. Hatzes, and D. N. C. Lin, Nature, 309, 333 (1984); A. P. Hatzes, F. G. Bridges, and D. N. C. Lin Mon. Not. R. Astr. Soc. 231, 1091 (1988); K. D. Supulver, G. F. G. Bridges, and D. N. C. Lin, ICARUS 113, 188 (1995).

[11] For small impact velocity the coefficient of restitution approaches 1, however, we should remark here that experiments have shown that for extremely slow impact the coefficient of restitution drops again because sticking forces begin to govern the interaction between the particles. See e.g. A. Hatzes, F. G. Bridges, D. N. C. Lin, S. Sachtjen, Icarus 89, 113 
(1991); F. G. Bridges, K. D. Supulver, D. N. C. Lin, R. Knight, and M. Zafra, preprint; J. Blum, M. Muench, Icarus 106, 151 (1993).

[12] K. L. Johnson, Contact Mechanics, Cambridge University Press, (Cambridge, 1985).

[13] Y. H. Pao, J. Applied Phys., 26, 1083 (1955).

[14] P. K. Haff, J. Fluid Mech. 134, 401 (1983).

[15] C. K. K. Lun and S. B. Savage, Acta Mechanica, 63, 15 (1986).

[16] S. E. Esipov and T. Pöschel, J. Stat. Phys. (in press).

[17] H. Hertz, J. f. reine und angewandte Physik, 92, 156 (1882); On the contact of rigid elastic solids and hardness, miscellaneous papers, McMillan, (London, 1886). 


\section{FIGURES}

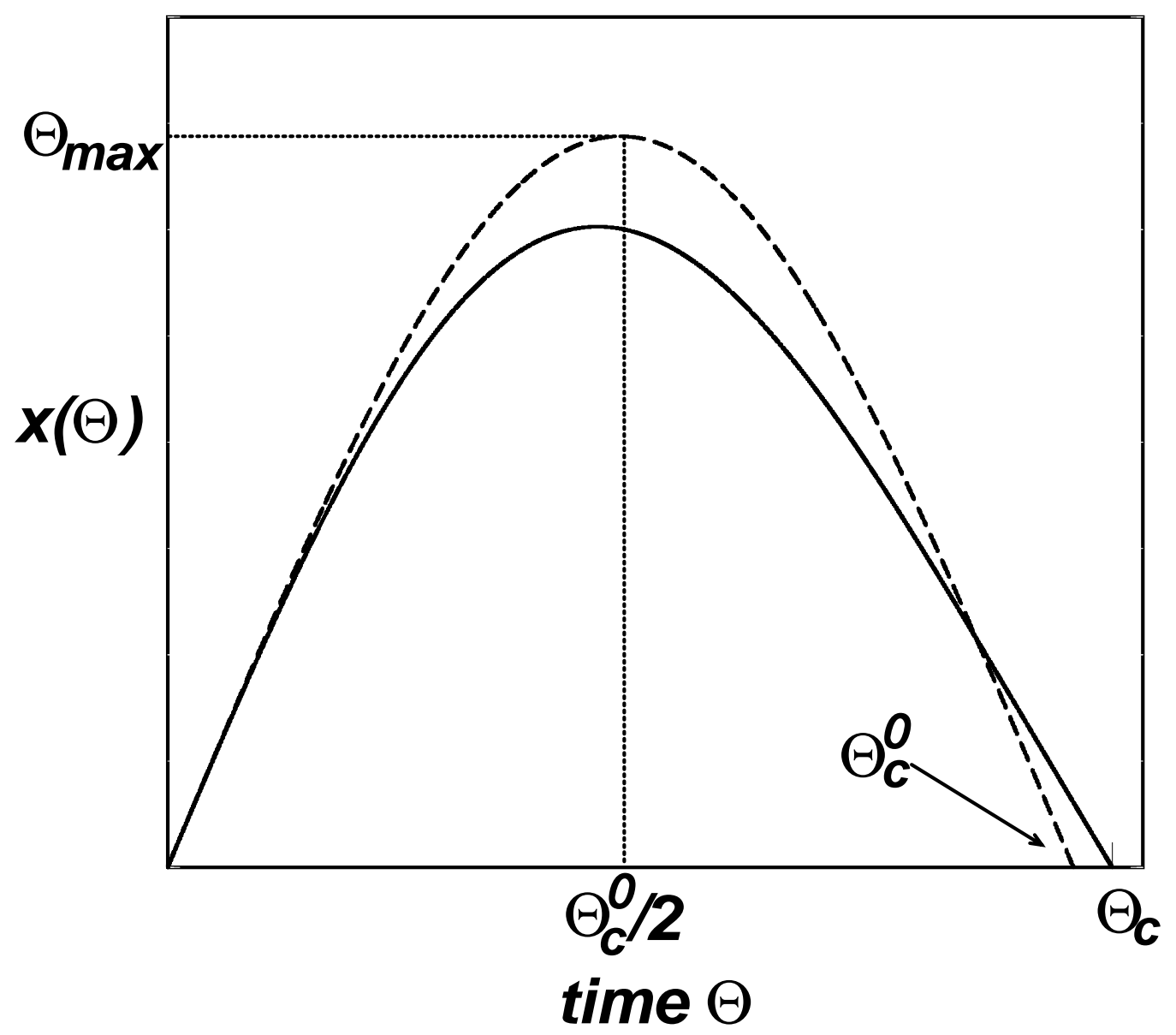

FIG. 1. The dynamics of the collision. The dashed line shows the (strictly symmetric) solution of the undamped collision. For the case of the damped motion (full line) the maximum penetration depth is achieved earlier whereas the duration of the collision is longer $\left(\Theta_{c}>\Theta_{c}^{0}\right)$. 


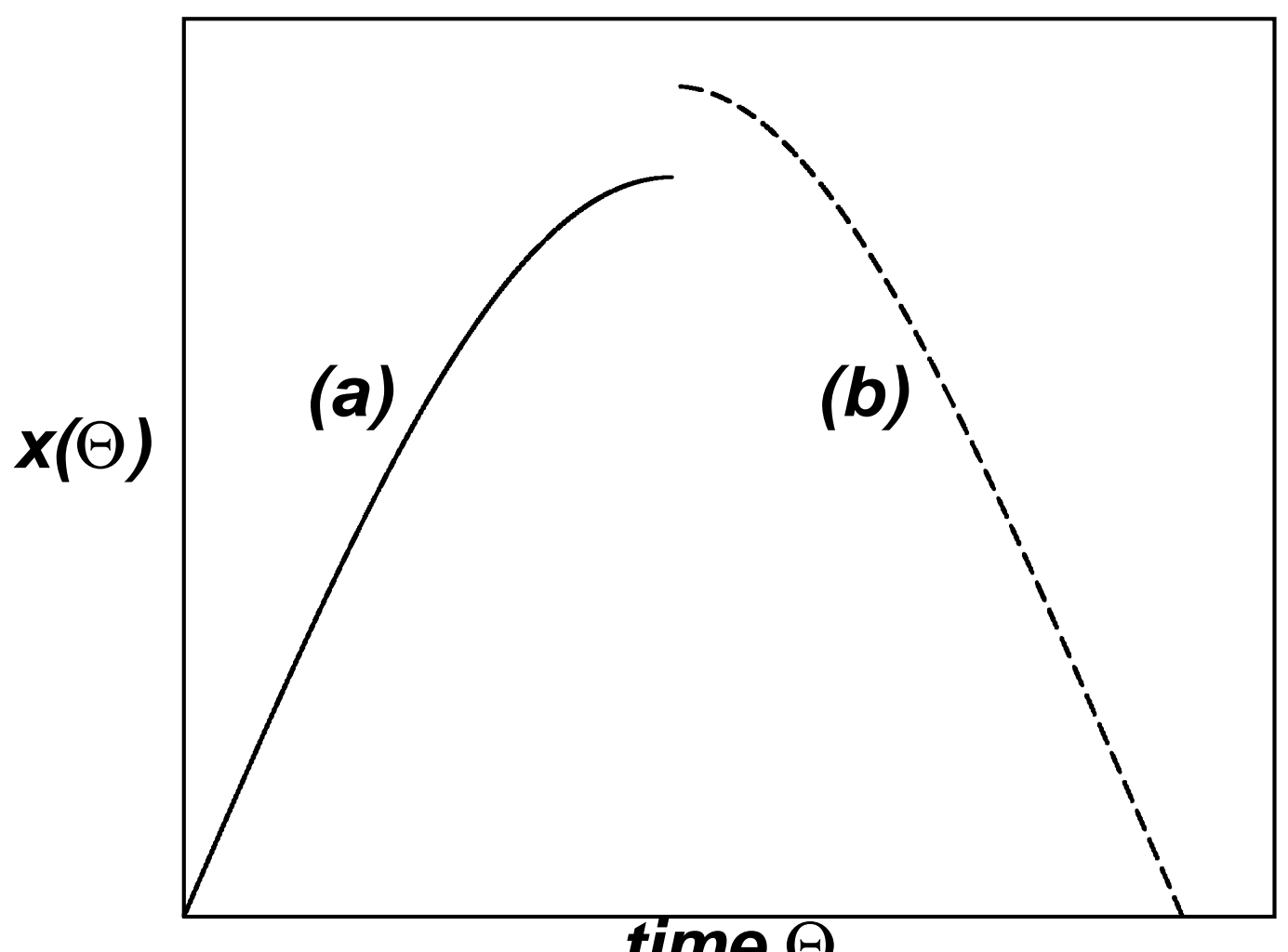

FIG. 2. Sketch of the calculation. The first part $(a) \Theta \in\left(0, \Theta_{m}\right)$ is calculated directly, for the other part $(b)$ we define the inverse collision where the particles start with velocity $v^{\prime}$ and velocity approaches zero at $\Theta=\Theta_{m}$. Both curves have to fit together smoothly. 


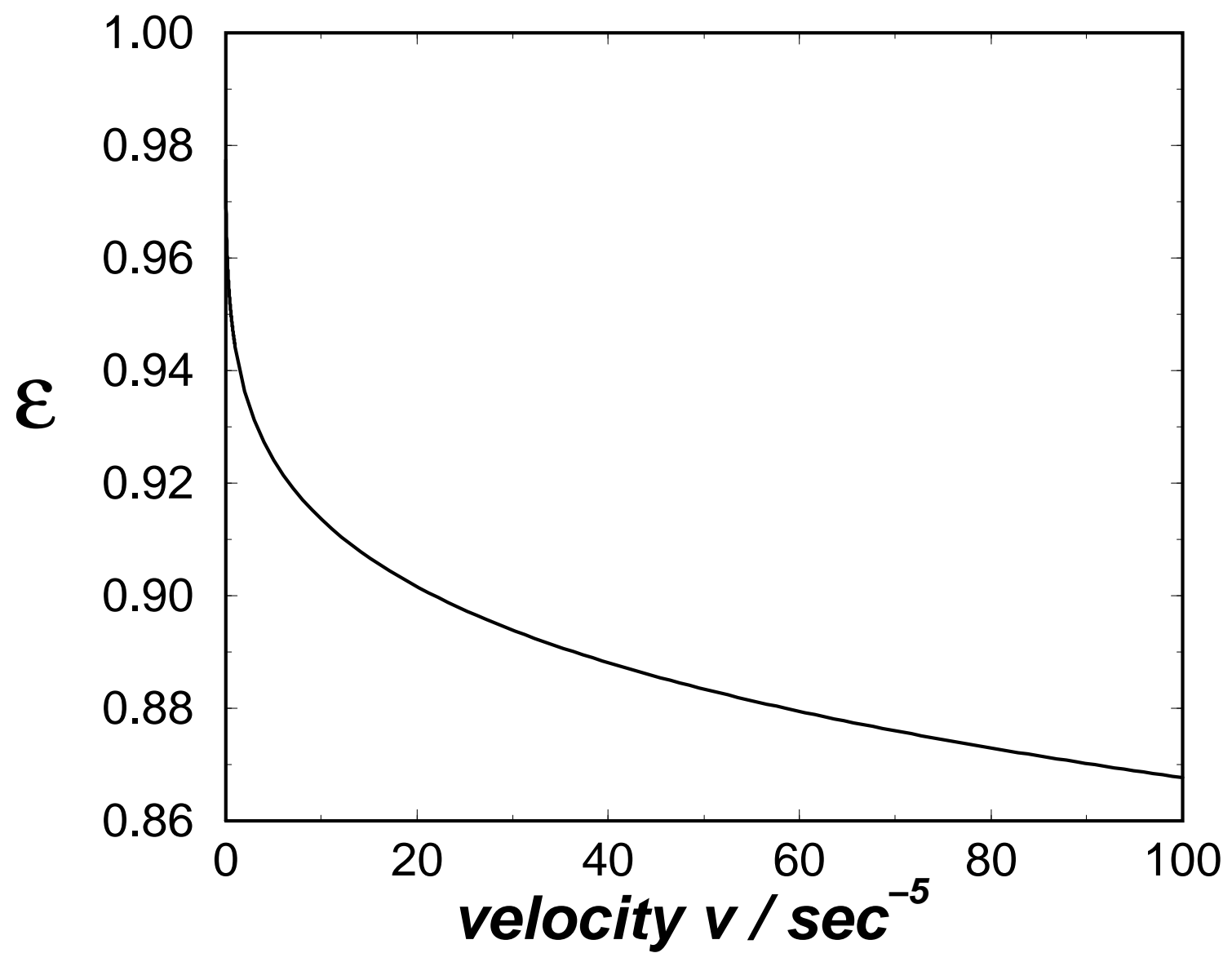

FIG. 3. The coefficient of restitution over impact velocity due to eq. (42). As expected for small relative velocity the particles collide almost elastically. The result of numerical integration of (6) coincides with the curve. Both curves cannot be distinguished in the plot. 